\title{
BOUNDARIES OF SEMILINEAR SPACES AND SEMIALGEBRAS
}

\author{
BY \\ R. E. WORTH
}

\section{ChAPTER 1. InTRODUCTION}

A natural generalization of the concepts of Banach space and Banach algebra is obtained by omitting the condition that the sets under addition form a group, substituting the condition that addition yield a semigroup. Of course, the scalars involved in scalar multiplication must be restricted, otherwise nothing has actually been changed. With the absence of subtraction, the norm becomes useless, and must be replaced by a metric satisfying special conditions.

F. F. Bonsall, S. Bourne, and E. J. Barbeau (see the bibliography), among others, have published results concerning this generalization. Bonsall and Barbeau take a semialgebra to be a subset of a Banach algebra which is closed under addition, multiplication, and scalar multiplication by nonnegative reals. Recall here the extensive work on convex cones in locally convex topological vector spaces (Schaefer's book is a good example)-see example (5) of this work. Bourne starts with a semiring, then concentrates on unital left $\Sigma$-semimodules with various restrictions and additional operations ( $\Sigma$ the nonnegative reals, invariant metric, etc.), among which is semialgebra as defined in this paper. The question arises here whether or not these generalizations are more a difference in point of view than a study of something different; that is, can one always consider a semialgebra, semilinear space, etc., as just a convex cone, semialgebra, etc. in a linear space or algebra. Algebraically, an embedding can be achieved-e.g., mimic the extension of the nonnegative integers to the integers. The real question is whether the metric or topology can be extended. Bourne [8] has something to say about extending norms; Keimel [14] considers the locally compact topological case. It might be said that we have carried some of Bourne's point of view over into a study of the kind of spaces Bonsall and Barbeau have in mind. However, this is not all we have done. We have introduced new concepts (boundary, interior, and linear independence) and studied them in enough detail to demonstrate that they are a significant addition to the literature on semialgebras. The entire field has many gaping holes which need to be filled before outstanding problems can be solved.

Presented to the Society, November 11, 1967 under the title The boundary of a semilinear space and January 25, 1969 under the title The boundary of a semi-algebra; received by the editors March 3, 1969 and, in revised form, May 21, 1969.

Copyright (C) 1970, American Mathematical Society 
In the body of this work we start by considering a semilinear space to be a set $S$ on which is given two operations, addition and scalar multiplication by nonnegative real numbers only, such that $S$ under addition is a commutative, cancellative semigroup with additive identity 0 . We then study, in turn, topological, metric, and complete metric semilinear spaces (called Banach semilinear spaces). A metric semilinear space $S$ has a metric $d$ which is invariant, that is, $d(\alpha x+y, \alpha z+y)$ $=\alpha d(x, z)$, for all $x, y, z$ in $S$, and $\alpha \geqq 0$. We then take a semialgebra to be a semilinear space on which is given an operation of (elementwise) multiplication under which $S$ is a semigroup, with the usual relationships between the three operations. We follow up by considering topological, metric, and Banach semialgebras.

Throughout, special attention is given to the boundary of a semilinear space, defined algebraically rather than topologically. Let $[x, y]$ denote the set of all $\alpha x+\beta y$, where $\alpha \geqq 0, \beta \geqq 0$, and $\alpha+\beta=1$. The boundary $\beta(S)$ of a semilinear space $S$ is the set of all $x$ in $S$ such that there exists $y$ in $S$ such that $x$ is not in $[y, z]$ for any $z \neq x$.

The interior $I(S)$ of a topological semilinear space $S$ is the union of all open sets $V$ such that if $U$ is open, $U \subset V$, and $x$ is in $S$, then $x+U$ is open. In a topological semilinear space $S$, we prove that $\beta(S)$ and $I(S)$ partition $S$, with $\beta(S)$ closed and $I(S)$ open.

One very useful result is that if $S$ is a Banach semilinear space and $x$ and $y$ are points of $S$ such that $z$ in $\beta(S)$ implies that $x$ is not in $[x+y, z]$, then $y$ has an additive inverse.

A subset $L$ of $\beta(S)$ is called a linear subspace of $\beta(S)$ if it is closed under addition and scalar multiplication. Suppose $L$ is a maximal linear subspace of the Banach semilinear space $S$. We denote by $y / x$ the set of all $v$ in $S$ such that $y$ is in $[x, v]$, and by $L y / x$ the closure of the set of all $z$ in $S$ such that $z$ is in $[u, v]$, for some $u$ in $L$ and $v$ in $y / x$.

We have the following results. Suppose the Banach semilinear space $S$ contains a maximal linear subspace $L$ and $x_{0}$ in $L, y_{0}$ not in $L$, such that $L y_{0} / x_{0}=S$. Then the $x_{0}$ corresponding to $y_{0}$ such that $L y_{0} / x_{0}=S$ is unique if and only if 0 is the only element of $S$ with additive inverse. If there exists $x_{0}$ in $L$ and $y_{0}$ not in $L$ such that $L y_{0} / x_{0}=S$, then there exists $z$ in $S$ such that $L z / 0=S$, where 0 is the additive identity.

When is there $z$ not in $L$ such that $L z / 0=S$ ? We prove that $L z / 0=S$ if and only if $z$ is in every maximal linear subspace of $\beta(S)$ distinct from $L$.

We say that linear combination $\alpha_{1} x_{1}+\cdots+\alpha_{n} x_{n}$ is reduced if $x_{i}+x_{j}=0$ implies that either $\alpha_{i}=0$ or $\alpha_{j}=0$. Every linear combination is equal to a reduced combination, and we assume that every combination has been so reduced. The points $x_{1}, x_{2}, \ldots, x_{n}$ are linearly independent if $\alpha_{1} x_{1}+\cdots+\alpha_{n} x_{n}=\beta_{1} x_{1}+\cdots+\beta_{n} x_{n}$ implies $\alpha_{i}=\beta_{i}, i=1, \ldots, n$. Note that this allows additive inverse of the $x_{i}$ to be included. We have the natural extensions to maximal linearly independent subsets and bases of semilinear spaces. Not all such spaces have a basis. 
We prove that if the Banach semilinear space $S$, with nonempty interior, has a basis $B$, then $B$ is not a subset of $\beta(S)$ if and only if every element of $\beta(S)$ has an additive inverse. Also, if $B$ is not a subset of $\beta(S)$, then exactly one point of $B$ is in $I(S)$.

Turning to semialgebras, we concentrate on ideals. The subset $M$ of the semialgebra $S$ is an ideal provided that $M$ is a semialgebra and $a$ in $M, x$ in $S$ imply $a x, x a$ in $M$. The ideal $M$ is nonassimilating if there is $x$ in $S$ such that $x+m$ is not in $M$, for any $m$ in $M$. Given an ideal $M$, we say that $x$ is congruent to $y$ modulo $M$ if there exist $m$ and $n$ in $M$ such that $x+m=y+n$, and write $x \equiv y(M)$. The relation " $\equiv$ " is an equivalence relation. We denote by $\bar{x}$ the equivalence class containing $x$.

One result we obtain is that if $M$ is a nonassimilating maximal ideal in a Banach semialgebra $S$ and there exists $x$ in $S$ such that $\bar{x} \cap \beta(S)$ is empty, then $M=\beta(S)$ and $M$ is the set of elements of $S$ which have additive inverses.

A slightly surprising result is the following. If $S$ is a Banach semialgebra with identity $e \in I(S)$ such that $d(e, 0)=1$, then $d(e, x)<1$ implies $x$ is regular. This theorem implies the well-known theorem from Banach algebras.

This work is contained in the author's doctoral dissertation submitted to the University of Georgia. He wishes to thank Professor John W. Jewett for his help and encouragement.

The author also wishes to thank the referee for his extremely perceptive and comprehensive scrutiny of this work.

\section{Chapter 2. Semilinear SPaces}

\section{Definitions.}

Definition 2.1. A semilinear space is a set $S$ on which is defined operations of addition $(+)$ and scalar multiplication by nonnegative real numbers (henceforth denoted by Greek letters) such that the following properties hold:

(a) $(S,+)$ is a commutative, cancellative semigroup with 0 ,

(b) $\alpha(x+y)=\alpha x+\alpha y$,

(c) $(\alpha+\beta) x=\alpha x+\beta x$,

(d) $\alpha(\beta x)=(\alpha \beta) x$,

(e) $1 \cdot x=x$ and $0 \cdot x=0$, where on the left 0 and 1 are real numbers and on the right 0 is the additive identity.

Definition 2.2. A topological semilinear space is a semilinear space $S$ on which is defined a Hausdorff topology such that addition is continuous from $S \times S$ into $S$ and scalar multiplication is continuous from $R^{+} \times S$ into $S$, where $R^{+}$ denotes the set of nonnegative real numbers.

Definition 2.3. A metric semilinear space is a semilinear space $S$ on which is defined a metric $d$ satisfying the additional condition $d(\alpha x+z, \alpha y+z)=\alpha d(x, y)$.

DEFINITION 2.4. A Banach semilinear space is a complete metric semilinear space. 
2. Examples.

(1) Any linear space is a semilinear space.

(2) Any linear topological space is a topological semilinear space.

(3) Any normed linear space is a metric semilinear space.

(4) Any Banach space is a Banach semilinear space.

(5) A convex cone in a topological linear space (Banach space) is a topological linear space (metric semilinear space).

(6) Let $E_{2}^{+}=\{(x, y): x \geqq 0, y \geqq 0\}$. With the usual addition and scalar multiplication and

$$
d\left(\left(x_{0}, y_{0}\right),\left(x_{1}, y_{1}\right)\right)=\max \left\{\left|x_{0}-x_{1}\right|,\left|y_{0}-y_{1}\right|\right\}
$$

$E_{2}^{+}$is a Banach semilinear space.

(7) Let $E_{2}^{r}=\{(x, y): x \geqq 0\}$. With operations and metric as in (6), $E_{2}^{r}$ is a Banach semilinear space.

(8) Let $S$ be the set of all sequences of complex (or real) numbers which converge to a nonnegative real number. With coordinatewise addition and scalar multiplication (by nonnegative reals), and metric $d(x, y)=\sup \left|x_{n}-y_{n}\right|, S$ is a Banach semilinear space. Or, let $S$ be the set of all bounded sequences with $\lim \inf x_{n} \geqq 0$.

(9) In (8), let $S$ be the set of all convergent sequences of complex numbers so that, if $z_{n} \rightarrow z$, then $\operatorname{Re}(z) \geqq 0$ and $\operatorname{Im}(z) \geqq 0$.

(10) Let $F$ be the set of all nonnegative functions defined on the integers which are nonzero at only a finite number of points. With the usual addition and scalar multiplication and sup metric, $F$ is a metric semilinear space which is not complete. An additional property will be brought out later.

CHAPTER 3. THE BOUNDARY AND INTERIOR

1. The set $I$. We first consider the set of elements of a semilinear space which have additive inverses, and its complement.

Definition 3.1. Given a semilinear space $S$, let $I=\left\{x \in S: x+x^{\prime}=0\right.$, for some $\left.x^{\prime} \in S\right\}$, and let $N=S-I$.

THEOREM 3.1. Let $S$ be a topological semilinear space, with $N$ nonempty. Then $N$ is dense in $S$.

Proof. Let $m_{0} \in N$ and $m \in I$. For $\alpha>0, \beta \geqq 0, \alpha+\beta=1$, we have $\alpha m_{0}+\beta m \in N$; for otherwise $\left(\alpha m_{0}+\beta m\right)+x=0$, for some $x \in S$, or $m_{0}+((\beta / \alpha) m+(1 / \alpha) x)=0$, which implies $m_{0} \in I$. Clearly, the sequence $\left\{(1 / n) m_{0}+(1-1 / n) m\right\}, n=1,2, \ldots$, of elements of $N$ converges to $m$. Thus each point of $I$ is a limit point of $N$, which shows that $N$ is dense.

THEOREM 3.2. If $S$ is a Banach semilinear space, then I is closed. Hence, if $N \neq \varnothing$, then $N$ is open and dense in $S$. 
Proof. Suppose $x_{0}$ is a limit point of $I$, so that there is a sequence $\left\{x_{n}\right\}$ of points of $I$ which converges to $x_{0}$. For each $n(=1,2, \ldots)$ there is $x_{n}^{\prime} \in S$ such that $x_{n}+x_{n}^{\prime}=0$.

Since $\left\{x_{n}\right\}$ converges, it is a Cauchy sequence. Now, using Definition 1.3, we have

$$
\begin{aligned}
d\left(x_{m}^{\prime}, x_{n}^{\prime}\right) & =d\left(x_{m}^{\prime}+x_{m}+x_{n}, x_{n}^{\prime}+x_{m}+x_{n}\right) \\
& =d\left(x_{n}+0, x_{m}+0\right)=d\left(x_{m}, x_{n}\right) .
\end{aligned}
$$

This implies that $\left\{x_{n}^{\prime}\right\}$ is also a Cauchy sequence. Since $S$ is complete, $\left\{x_{n}^{\prime}\right\}$ converges, say to $x_{0}^{\prime}$. Now,

$$
\begin{aligned}
d\left(x_{0}+x_{0}^{\prime}, 0\right) & =d\left(x_{0}+x_{0}^{\prime}, x_{n}+x_{n}^{\prime}\right) \\
& \leqq d\left(x_{0}+x_{0}^{\prime}, x_{0}+x_{n}^{\prime}\right)+d\left(x_{0}+x_{n}^{\prime}, x_{n}+x_{n}^{\prime}\right) \\
& =d\left(x_{0}^{\prime}, x_{n}^{\prime}\right)+d\left(s_{0}, x_{n}\right) .
\end{aligned}
$$

Since the last expression can be made as small as desired, we must have $d\left(x_{0}+x_{0}^{\prime}, 0\right)$ $=0$. Therefore $x_{0}+x_{0}^{\prime}=0, x_{0} \in I$, and $I$ is closed.

We conclude this section with a curious connection between $I$ and open neighborhoods of 0 .

Consider first the right half-plane, example (7). In this, $I$ is the $y$-axis. Let $V$ be an open ball in $E_{2}^{r}$ containing 0 (a semicircle with left edge included). If $x \in I$, then $x+V=\{x+v: v \in V\}$ is seen to be open. However, if $x \notin I$, then $x+V$ contains its left edge and will not be open. This is not a coincidence.

THEOREM 3.3. Let $S$ be a topological semilinear space and $V$ an open neighborhood of 0 . Then $x \in I$ if and only only if $x+V$ is open.

Proof. Suppose $x \in I$. Then $x+x^{\prime}=0$, for some $x^{\prime}$. The mapping $y \rightarrow y+x^{\prime}$ is clearly continuous, and $x+V$ is the inverse image of $V$. Hence $x+V$ is open.

Suppose $x+V$ is open. Since the sequence $\{(1-1 / n) x\}$ converges to $x$, we must have $(1-1 / n) x \in x+V$, for some $n$, since $x+0 \in x+V$. Then there is $x_{0} \in V$ such that $(1-1 / n) x=x+x_{0}$. From $x+x_{0}=(1-1 / n) x+(1 / n) x+x_{0}$ and cancellativity of addition, we have $(1 / n) x+x_{0}=0$ and $x+n x_{0}=0$. Therefore $x \in I$.

2. Boundary and interior. Let $S$ be a topological semilinear space. We assume $N \neq \varnothing$, so that $S$ is not a linear space.

Given $x, y \in S$, let

$$
[x, y]=\{\alpha x+\beta y: \alpha \geqq 0, \beta \geqq 0, \alpha+\beta=1\} .
$$

Definition 3.2. The boundary $\beta(S)$ of $S$ is the set of all $x \in S$ such that there exists $x_{0} \in S$ such that $x \notin\left[x_{0}, x^{\prime}\right]$, for $x^{\prime} \neq x$.

Definition 3.3. The interior $I(S)$ of $S$ is the union of all open sets $V$ such that if $U$ is open, $U \subset V$, and $x \in S$, then $x+U$ is open.

Note that it immediately follows that $I(S)$ is open.

THEOREM 3.4. (a) If $V$ is a nonempty open subset of $I(S)$ and $x_{0} \in S, \alpha>0$, then $x_{0}+V$ and $\alpha V=\{\alpha v: v \in S\}$ are open subsets of $I(S)$. 
(b) If $x \in I(S), y \in S$ and $\alpha>0, \beta \geqq 0, \alpha+\beta=1$, then $z=\alpha x+\beta y \in I(S)$.

(c) $I(S)$ is dense in $S$ if $I(S) \neq \varnothing$.

(d) $\beta(S) \subset S-I(S)$; if $I(S) \neq \varnothing, \beta(S)=S-I(S)$.

(e) $I \subset \beta(S)$ and $I(S) \subset N$.

Proof. (a) We show that $x_{0}+V$ is open. Let $x=x_{0}+v \in x_{0}+V$, where $v \in V$. Since $v \in I(S)$, there is open $V_{0}$ containing $v$ such that $x_{0}+U_{0}$ is open for $U_{0}$ an open subset of $V_{0}$. Now $V_{0} \cap V$ is an open subset of $V_{0}$, so that $x_{0}+V_{0} \cap V$ is open. Since $x_{0}+v \in x_{0}+V_{0} \cap V \subset x_{0}+V$, it follows that $x_{0}+V$ is open.

Now let $z \in S$. Then $\left(z+x_{0}\right)+V$ is open, so that $z+\left(x_{0}+V\right)$ is open. This implies that $x_{0}+V \subset I(S)$.

$\alpha V$ is open, since under the continuous mapping $y \rightarrow(1 / \alpha) y, \alpha V$ is the inverse image of the open set $V$.

Given $x \in S, x+V$ is open. As in the last argument, $\alpha(x+V)=\alpha x+\alpha V$ is open. Given $u \in S$, let $x=(1 / \alpha) u$. Then $u+\alpha V$ is open. It follows from Definition 3.3 that $\alpha V \subset I(S)$.

(b) We have $x+(\beta / \alpha) y=(1 / \alpha) z$. Since $x \in I(S)$, there exists an open neighborhood $V$ of $x$ such that $x_{0}+V$ is open for any $x_{0} \in S$. Then $(\beta / \alpha) y+V$, and hence $\alpha((\beta / \alpha) y+V)=\beta y+\alpha V$, are open subsets of $I(S)$. Since $\alpha x \in \alpha V$, we have $z=\beta y$ $+\alpha x \in I(S)$.

(c) follows immediately from (b).

(d) Let $x \in \beta(S)$. Then there exists $x_{0}$ such that $x \notin\left[x_{0}, x^{\prime}\right]$ for $x^{\prime} \neq x$. Suppose $x \in I(S)$. Then there is an open neighborhood $V$ of $x$ such that $x_{0}+V$ is open. Since addition is continuous, there must exist neighborhoods $V_{1}$ and $V_{2}$ of $x_{0}$ and $x$, respectively, so that $y \in V_{1}$ and $z \in V_{2}$ imply $y+z$ are in the neighborhood $x_{0}+V$ of $x_{0}+x$. Since scalar multiplication is continuous, there is $0<\beta<1$ such that $\beta x_{0} \in V_{1}$. This with $x \in V_{2}$ imply $(1-\beta) x+\beta\left(x+x_{0}\right)=x+\beta x_{0} \in x_{0}+V$. Then there exists $v \in V$ so that

$$
\begin{aligned}
x+\beta x_{0} & =x_{0}+v, \\
x & =(1-\beta) x_{0}+v, \\
x & =(1-\beta) x_{0}+\beta((1 / \beta) v) .
\end{aligned}
$$

Clearly, $x \neq(1 / \beta) v$. Thus we have $x \in\left[x_{0},(1 / \beta) v\right]$, with $x \neq x_{0}, x \neq(1 / \beta) v$, which contradicts the choice of $x_{0}$. Therefore $x \notin I(S)$, i.e. $x \in S-I(S)$. This means $\beta(S) \subset S-I(S)$.

$0 \in S-I(S)$, for if $0 \notin I(S)$, Theorem 3.3 and Definition 3.3 would imply $I=S$, contrary to our assumption that $N \neq \varnothing$. Thus $S-I(S) \neq \varnothing$.

Let $x \in S-I(S)$. Suppose $I(S) \neq \varnothing$, and let $z \in I(S)$. Suppose there exists $x^{\prime} \neq x$ such that $x \in\left[z, x^{\prime}\right]$. Then $x=\alpha z+\beta x^{\prime}$, with $\alpha>0, \beta>0$, and $\alpha+\beta=1$. But, by (b), $x \in I(S)$. Hence, $x^{\prime} \neq x$ implies $x \notin\left[z, x^{\prime}\right]$. By Definition 3.2, $x \in \beta(S)$, so that we have $S-I(S) \subset \beta(S)$. Hence $\beta(S)=S-I(S)$.

(e) Suppose there exists $x \in I$ such that $x \in I(S)$. Then, by (d) and Definition 3.2, given $y \neq x$, there is $y^{\prime} \neq x$ such that $x=\alpha y+\beta y^{\prime}$, with $\alpha>0, \beta>0, \alpha+\beta=1$. 
Since $x \in I$, we have $x+x^{\prime}=0$ for some $x^{\prime} \in S$. We have then $\alpha y+\beta y^{\prime}+x^{\prime}=0$, $y+\left((\beta / \alpha) y^{\prime}+(1 / \alpha) x^{\prime}\right)=0$. Thus $y \in I$, which implies that $S=I$. This contradicts $N \neq \varnothing$. Therefore $I \subset \beta(S)$.

$I(S) \subset N$ follows from $I \subset \beta(S)=S-I(S)$ and $N=S-I$.

This completes the proof of the theorem.

Since $0 \in I \subset \beta(S), \beta(S)$ is always nonempty. After considering some of the more trivial examples, one might conjecture that $I(S)$ is always nonempty. This is not so. Consider example (10). Let $f \in F$. There is an integer $n_{0}$ such that $f\left(n_{0}\right)=0$. Let $f_{0}$ be the element of $F$ which is 1 at $n_{0}$ and 0 at $n \neq n_{0}$. Suppose there is $f^{\prime} \in F$ such that $f \in\left[f_{0}, f^{\prime}\right]$ and $f^{\prime} \neq f$. Then $\alpha f_{0}+\beta f^{\prime}=f$, where $\alpha>0, \beta>0$, and $\alpha+\beta=1$. Now $\alpha f_{0}\left(n_{0}\right)+\beta f^{\prime}\left(n_{0}\right)=f\left(n_{0}\right)=-\alpha / \beta$. But $f^{\prime}\left(n_{0}\right)$ must be nonnegative. This is a contradiction. By Definition 3.2, $f \in \beta(F)$, i.e. $F=\beta(F)$. Since $\beta(F) \subset F-I(F)$, by Theorem 3.4(d), we must have $I(F)=\varnothing$.

The author wishes to thank the referee for the following intriguing example. Let $S$ be all nonnegative sequences with $x_{1} \geqq x_{i}$, for $i=1,2, \ldots$, with the topology of pointwise convergence. Then $\left(1, \frac{1}{2}, \frac{1}{2}, \frac{1}{2}, \ldots\right)$ is not in $\beta(S)$ but $I(S)=\varnothing$.

\section{Chapter 4. GeOMetry in SEMILINEAR SPACES}

\section{Some miscellaneous theorems.}

THEOREM 4.1. Let $S$ be a metric semilinear space.

(a) If $\lim a_{n}=a, a_{n} \in S$, and $\lim \alpha_{n}=\alpha$, then $\lim \alpha_{n} a_{n}=\alpha a$.

(b) If $a_{n}+b_{n}=c_{n}$ and any two of $\lim a_{n}, \lim b_{n}$, and $\lim c_{n}$ exist, then so does the third and $\lim a_{n}+\lim b_{n}=\lim c_{n}$.

Proof. Straightforward use of Definition 2.3.

THEOREM 4.2. Let $S$ be as in 4.1.

(a) $d(\alpha x, \beta x)=|\alpha-\beta| d(x, 0)$.

(b) If $\alpha x+\beta y=z$ and $\alpha+\beta=1$, then $\alpha=d(y, z) / d(x, y)$.

Proof of (b). $d(x, y)=(1 / \alpha) d(\alpha x, \alpha y)=(1 / \alpha) d(\alpha x+\beta y, \alpha y+\beta y)=(1 / \alpha) d(z, y)$.

THEOREM 4.3. Suppose $S$ is a Banach semilinear space and $x$ and $y$ are points of $S$ such that if $x_{0} \in \beta(S)$, then $x \notin\left[x+y, x_{0}\right]$. Then $y \in I$.

Proof. Two facts are needed:

(1) If $\alpha>0, \beta>0, \alpha+\beta=1$, and $\alpha z+\beta(x+y)=x$. then $d(x, z)=(\beta / \alpha) d(y, 0)$.

For $z+(\beta / \alpha) y=x$, and $d(x, z)=d(x+(\beta / \alpha) y, z+(\beta / \alpha) y)=d(x+(\beta / \alpha) y, x)=$ $d((\beta / \alpha) y, 0)=(\beta / \alpha) d(y, 0)$.

(2) If, in addition to the conditions of (1), we have $\alpha_{0} z_{0}+\beta_{0}(x+y)=x, \alpha_{0}>0$, $\beta_{0}>0, \alpha_{0}+\beta_{0}=1$, then $d\left(z, z_{0}\right)=\left|\beta_{0} / \alpha_{0}-\beta / \alpha\right| d(y, 0)$.

For

$$
\begin{aligned}
d\left(z, z_{0}\right) & =d\left(z+\left(\beta_{0} / \alpha_{0}\right) y+(\beta / \alpha) y, z_{0}+\left(\beta_{0} / \alpha_{0}\right) y+(\beta / \alpha) y\right) \\
& =d\left(x+\left(\beta_{0} / \alpha_{0}\right) y, x+(\beta / \alpha) y\right)=d\left(\left(\beta_{0} / \alpha_{0}\right) y,(\beta / \alpha) y\right) \\
& =\left|\beta_{0} / \alpha_{0}-\beta / \alpha\right| d(y, 0),
\end{aligned}
$$

by Definition 2.3 and Theorem $4.2(\mathrm{a})$. 
Suppose now that $\{d(z, x): x \in[x+y, z]\}$ is bounded, and let $\gamma$ be its least upper bound. We cannot have $y=0$, for then $x \in[x+y, z]$ for any $z \in S$. We cannot have $\gamma=0$, for then if $z \neq x$, we have $x \notin[x+y, z]$, which by Definition 3.2 implies $x \in \beta(S)$, contradicting the hypothesis. Therefore $d(y, 0)>0$ and $\gamma>0$.

There exists a sequence $\left\{z_{n}\right\}$ such that $\gamma-1 / n<d\left(z_{n}, x\right) \leqq \gamma$ and $x \in\left[x+y, z_{n}\right]$ for each $n$. For each $n$, there exist $\alpha_{n}>0, \beta_{n}>0$, with $\alpha_{n}+\beta_{n}=1$, such that $\alpha_{n} z_{n}$ $+\beta_{n}(x+y)=x$. By $(1)$ above $d\left(z_{n}, x\right)=\left(\beta_{n} / \alpha_{n}\right) d(y, 0)$. Then $\gamma-1 / n<\left(\beta_{n} / \alpha_{n}\right) d(y, 0)$ $\leqq \gamma$. This implies that $\left(\beta_{n} / \alpha_{n}\right) d(y, 0)$ converges to $\gamma$, so that $\alpha_{n}$ converges to $1 /[\gamma / d(m, 0)+1]=\alpha>0$, and $\beta_{n}$ converges to $1-\alpha>0$. Since

$$
d\left(z_{n}, z_{k}\right)=\left|\beta_{n} / \alpha_{n}-\beta_{k} / \alpha_{k}\right| d(y, 0),
$$

by (2), and $\left\{\beta_{n} / \alpha_{n}\right\}$ converges, we have that $d\left(z_{n}, z_{k}\right)$ converges to 0 . Thus $\left\{z_{n}\right\}$ is a Cauchy sequence and hence converges, say to $z$. We have $z_{n} \rightarrow z, \alpha_{n} \rightarrow \alpha$, and $\beta_{n} \rightarrow \beta$, so, by Theorem 3.1, $\alpha_{n} z_{n}+\beta_{n}(x+y)$ converges to $\alpha z+\beta(x+y)$. Since $\alpha_{n} z_{n}+\beta_{n}(x+y)=x, \alpha z+\beta(x+y)=x$. Note that

$$
d(z, x)=(\beta / \alpha) d(y, 0)=(1 / \alpha-1) d(y, 0)=\gamma .
$$

From the hypotheses, we see that $z \notin \beta(S)$, so there must exist $z^{\prime} \neq z, \alpha^{\prime}>0$, $\beta^{\prime}>0$, with $\alpha^{\prime}+\beta^{\prime}=1$, such that $\alpha^{\prime} x+\beta^{\prime} z^{\prime}=z$. This, with $\alpha z+\beta(x+y)=x$, implies that $\left(\alpha \beta^{\prime} /\left(1-\alpha \alpha^{\prime}\right)\right) z^{\prime}+\left(\beta /\left(1-\alpha \alpha^{\prime}\right)\right)(x+y)=x$, where $\alpha \beta^{\prime} /\left(1-\alpha \alpha^{\prime}\right)>0, \beta /\left(1-\alpha \alpha^{\prime}\right)>0$, and $\alpha \beta^{\prime} /\left(1-\alpha \alpha^{\prime}\right)+\beta /\left(1-\alpha \alpha^{\prime}\right)=1$. By (1),

$$
d\left(z^{\prime}, x\right)=\frac{\beta}{\alpha \beta^{\prime}} d(y, 0)=\frac{1-\alpha}{\alpha} \frac{1}{\beta^{\prime}} d(y, 0)=\left(\frac{1}{\alpha}-1\right) \frac{1}{\beta^{\prime}} d(y, 0)=\gamma\left(\frac{1}{\beta^{\prime}}\right)>\gamma .
$$

This contradicts the choice of $\gamma$. Thus we have that $\{d(z, x): x \in[x+y, z]\}$ is unbounded.

Given a positive integer $n$, there exists $z_{n}$ such that $d\left(z_{n}, x\right) \geqq n$ and $x \in$ $\left[x+y, z_{n}\right]$, i.e. there exist $\alpha_{n}>0, \beta_{n}>0$, with $\alpha_{n}+\beta_{n}=1$ such that $\alpha_{n} z_{n}+\beta_{n}(x+y)=x$. Now, by (1) $d\left(z_{n}, x\right)=\left(\beta_{n} / \alpha_{n}\right) d(y, 0)$. Let $y_{n}=z_{n} / d\left(z_{n}, x\right)$. Then $\alpha_{n} z_{n}+\beta_{n}(x+y)=x$ gives

and

$$
\alpha_{n} z_{n}+\beta_{n} y=\alpha_{n} x, \quad z_{n}+\frac{\beta_{n}}{\alpha_{n}} y=x, \quad y_{n}+\frac{\beta_{n}}{\alpha_{n}} \frac{1}{d\left(z_{n}, x\right)} y=\frac{1}{d\left(z_{n}, x\right)} x
$$

$$
y_{n}+\frac{1}{d(y, 0)} y=\frac{1}{d\left(z_{n}, x\right)} x,
$$

since $d\left(z_{n}, x\right)=\left(\beta_{n} / \alpha_{n}\right) d(y, 0)$. Since $1 / d\left(z_{n}, x\right)$ converges to 0 , by Theorem $4.1(\mathrm{~b})$ $y_{n}$ converges, say to $y_{0}$, and $y_{0}+(1 / d(y, 0)) y=0$, or $y+d(y, 0) y_{0}=0$. Therefore $y$ has an additive inverse and hence belongs to $I$.

\section{Maximal linear subspaces.}

Definition 4.1. If $S$ is a topological semilinear space, then the subset $L$ of $\beta(S)$ will be referred to as a maximal linear subspace of $\beta(S)$ if $L$ is a semilinear space which is not contained properly in any other semilinear subspace of $\beta(S)$. 
If $I(S) \neq \varnothing$, then Theorem 2.4 (d) and the fact that $I(S)$ is open imply that $\beta(S)$ is closed, and hence every maximal linear subspace of $\beta(S)$ is closed.

THEOREM 4.4. If $L$ is a maximal linear subspace of $\beta(S)$, then $I \subset L$.

Proof. If $x \in I$ and $y \in \beta(S)$, then $x+y \in \beta(S)$, for otherwise, by Theorem 3.4(a) and (b), $\frac{1}{2} y=\frac{1}{2}(-x)+\frac{1}{2}(x+y) \in I(S)$, so $y \in I(S)$, which contradicts Theorem 3.4 (d).

Suppose $x \in I$, and let $L^{\prime}$ be $\{\alpha x+\beta y: y \in L, \alpha \geqq 0, \beta \geqq 0\}$. Clearly $L^{\prime}$ is a semilinear space. For any $\alpha, \beta$ and $y \in L, \alpha x \in I$ and $\beta y \in \beta(S)$, so that by the above, $\alpha x+\beta y$. Then $L^{\prime} \subset \beta(S)$. Clearly, $L \subset L^{\prime}$. Since $L$ is maximal, $1=L^{\prime}$, so that $x=1 x+0 y \in L$. We conclude that $I \subset L$.

THEOREM 4.5. If $L$ is a maximal linear subspace of $\beta(S)$ and $x \notin L$, then $x+y \notin L$, for any $y \in L$.

Proof. Suppose there is $y \in L$ such that $x+y \in L$. If $x \in I(S)$, then $\frac{1}{2} x+\frac{1}{2} y \in I(S)$, by Theorem 3.4(b), and $2\left(\frac{1}{2} x+\frac{1}{2} y\right)=x+y \in I(S)$, by Theorem 3.4(a). But $x+y$ $\in L \subset \beta(S)$, which contradicts Theorem 3.4(d). Thus in case $x \in I(S), x+y \notin L$.

Suppose $x \in \beta(S)$. Then $\alpha x+\beta z \in \beta(S)$, for $\alpha \geqq 0, \beta \geqq 0$, and $z \in L$, for otherwise, if $\alpha x+\beta z \in I(S)$, then, by Theorem 3.4(b), $\alpha x+\beta z+a y \in I(S)$, so that $\alpha(x+y)+\beta z$ $\in I(S)$. But $\alpha(x+y)+\beta z \in L$, which is a contradiction. As in the concluding argument of Theorem 4.4, $x$ must be in $L$. This contradiction establishes the theorem.

Corollary. If $x, y \in L$ and $x \in[z, y]$, then $z \in L$.

Proof. Suppose $\alpha z+\beta y=x, \alpha>0$, with $\alpha+\beta=1$. Then $z+(\beta / \alpha) y=(1 / \alpha) x$, and $(1 / \alpha) x,(\beta / \alpha) y \in L$. By the theorem, if $z \notin L, z+(\beta / \alpha) y \notin L$.

3. The degeneracy of $I$. In this section we will consider only Banach semilinear spaces $S$ with nonempty interior.

Definition 4.2. Given $x, y \in S$, let $y / x$ denote the set of all $v \in S$ such that $y \in[x, v]$.

Definition 4.3. Given a maximal linear subspace $L$ of $\beta(S)$, let $O y / x$ denote the set of all $z \in S$ such that $z \in[u, v]$, for some $u \in L$ and $v \in y / x$. Let $L y / x$ denote the closure of $O y / x$.

EXAMPLES. Consider example (6), the first quadrant. Let $y=(1,1), x=(0,1)$, and $L$ be the maximal linear subspace of $\beta\left(E_{2}^{+}\right)$consisting of the $y$-axis. Then $L y / x=E_{2}^{+}$. Next consider example (7), the right half-plane. With $y$ and $L$ as above, we have that $L y / x=E_{2}^{r}$, for any $x \in L$. Note that in the first case the $x$ corresponding to $y=(1,1)$ is unique. Note also that in the first case $I=\{0\}$ and in the second case $I \neq\{0\}$.

Such examples can be found in infinite dimensional cases-for instance, example (8).

The major result of this section will be

THEOREM 4.6. Suppose $S$ contains a maximal linear subspace $L$ of $\beta(S)$ and 
$x_{0} \in L, y_{0} \in S-L$ such that $L y_{0} / x_{0}=S$. Then $I=\{0\}$ if and only if the $x_{0}$ such that $L y_{0} / x_{0}=S$ is unique.

The proof will be delayed until later.

THEOREM 4.7. If $L y_{0} / x_{0}=S$, then $y_{0} / x_{0}$ is unbounded.

Proof. Clearly, $y_{0} / x_{0}$ is unbounded if and only if $\left\{d\left(v, x_{0}\right): v \in y_{0} / x_{0}\right\}$ is unbounded.

Suppose $y_{0} / x_{0}$ is bounded, and let $\gamma_{0}=\operatorname{lub}\left\{d\left(v, x_{0}\right): v \in y_{0} / x_{0}\right\}$. If $\gamma_{0}=0$, then $x_{0} \in y_{0} / x_{0}$, which implies $x_{0}=y_{0}$, contradicting $y_{0} \in S-L$. Thus $\gamma_{0}>0$.

Let $\left\{v_{n}\right\} \subset y_{0} / x_{0}$ such that $\gamma_{0}-1 / n<d\left(v_{n}, x_{0}\right) \leqq \gamma_{0}$. Then there exist $\left\{\alpha_{n}\right\},\left\{\beta_{n}\right\}$, with $\alpha_{n}+\beta_{n}=1$, such that $\alpha_{n} v_{n}+\beta_{n} x_{0}=y_{0}$. By Theorem 4.2(b), $d\left(v_{n}, x_{0}\right)=$ $\left(1 / \alpha_{n}\right) d\left(x_{0} y_{0}\right)$. Then $\gamma_{0}-1 / \alpha_{n}<\left(1 / \alpha_{n}\right) d\left(x_{0} y_{0}\right) \leqq \gamma_{0}$, which implies that $\alpha_{n}$ converges to a nonzero number, say $\alpha_{0}$. Also $\gamma_{0}=\left(1 / \alpha_{0}\right) d\left(x_{0}, y_{0}\right)$. Also, $\beta_{n}$ converges to $1-\alpha_{0}$ $=\beta_{0}$.

Now $v_{n}+\left(\beta_{n} / \alpha_{n}\right) x_{0}=\left(1 / \alpha_{n}\right) y_{0}$. Since $\lim \left(\beta_{n} / \alpha_{n}\right) x_{0}=\left(\beta_{0} / \alpha_{0}\right) x_{0}$ and $\lim \left(1 / \alpha_{n}\right) y_{0}$ $=\left(1 / \alpha_{0}\right) y_{0}$ by Theorem 4.1(b) $v_{n}$ must converge, say to $v_{0}$. We have then $\alpha_{0} v_{0}+\beta_{0} x_{0}$ $=y_{0}$, with $\alpha_{0}+\beta_{0}=1$, i.e. $v_{0} \in y_{0} / x_{0}$.

We show that $y_{0} / x_{0}=\left[y_{0}, v_{0}\right]$. Clearly $\left[y_{0}, v_{0}\right] \subset y_{0} / x_{0}$. Let $v \in y_{0} / x_{0}$. Then $\alpha v+\beta x_{0}$ $=y_{0}$, where $\alpha+\beta=1$ and $\alpha>0$. We have $\alpha v+\beta x_{0}=\alpha_{0} v_{0}+\beta_{0} x_{0}$. Since $(1 / \alpha) d\left(x_{0}, y_{0}\right)$ $=d\left(v, x_{0}\right) \leqq \gamma_{0}=\left(1 / \alpha_{0}\right) d\left(x_{0}, y_{0}\right)$ we have $\alpha_{0} \leqq \alpha$ and $\beta \leqq \beta_{0}$. Then

$$
\begin{aligned}
v & =\frac{\alpha_{0}}{\alpha} v_{0}+\frac{\left(\beta_{0}-\beta_{0}\right)}{\alpha} x_{0}=\frac{\alpha_{0} \beta_{0}}{\alpha \beta_{0}} v_{0}+\frac{\left(\beta_{0}-\beta\right) \beta_{0}}{\alpha \beta_{0}} x_{0} \\
& =\frac{\beta \alpha_{0}}{\alpha \beta_{0}} v_{0}+\frac{\left(\beta_{0}-\beta\right) \alpha_{0}}{\alpha \beta_{0}} v_{0}+\frac{\left(\beta_{0}-\beta\right) \beta_{0}}{\alpha \beta_{0}} x_{0} \\
& =\frac{\beta \alpha_{0}}{\alpha \beta_{0}} v_{0}+\frac{\beta_{0}-\beta}{\alpha \beta_{0}}\left(\alpha_{0} v_{0}+\beta_{0} x_{0}\right)=\frac{\beta \alpha_{0}}{\alpha \beta_{0}} v_{0}+\frac{\beta_{0}-\beta}{\alpha \beta_{0}} y_{0}
\end{aligned}
$$

where

$$
\frac{\beta \alpha_{0}}{\alpha \beta_{0}}+\frac{\beta_{0}-\beta}{\alpha \beta_{0}}=1
$$

Hence $v \in\left[y_{0}, v_{0}\right]$ so that $y_{0} / x_{0}=\left[y_{0}, v_{0}\right]$.

Since $L y_{0} / x_{0}=S$, we have $2 v_{0} \in L y_{0} / x_{0}$, so there exists $\left\{z_{n}\right\}$ such that $z_{n} \in O y_{0} / x_{0}$ and $z_{n}$ converges to $2 v_{0}$. There exist $\left\{\alpha_{n}^{\prime}\right\},\left\{\beta_{n}^{\prime}\right\}$, with $\alpha_{n}^{\prime}+\beta_{n}^{\prime}=1$, such that $z_{n}=$ $\alpha_{n}^{\prime} u_{n}+\beta_{n}^{\prime} v_{n}$, where $u_{n}^{\prime} \in L$ and $v_{n}^{\prime} \in y_{0} / x_{0}$. Since $y_{0} / x_{0}=\left[y_{0}, v_{0}\right]$, there exist $\alpha_{n}^{\prime \prime}, \beta_{n}^{\prime \prime}$, with $\alpha_{n}^{\prime \prime}+\beta_{n}^{\prime \prime}=1$, such that $v_{n}^{\prime}=\alpha_{n}^{\prime \prime} v_{0}+\beta_{n}^{\prime \prime} x_{0}$.

Substituting, we get $z_{n}=\alpha_{n}^{\prime} u_{n}+\beta_{n}^{\prime} \alpha_{n}^{\prime \prime} v_{0}+\beta_{n}^{\prime} \beta_{n}^{\prime \prime} x_{0}$. Since $\left\{\beta_{n}^{\prime} \alpha_{n}^{\prime \prime}\right\}$ and $\left\{\beta_{n}^{\prime} \beta_{n}^{\prime \prime}\right\}$ are bounded above by 1 (and below by 0 ), some subsequences of each with common subscripts must converge. Since $z_{n}$ converges (hence any subsequence), by Theorem 4.1 the corresponding subsequence of $\alpha_{n}^{\prime} u_{n}$ must converge. Combining these results, we have $2 v_{0}=u_{1}+\gamma v_{0}+\gamma_{0} x_{0}$, where $u_{1} \in L$ ( $L$ is closed) and $\gamma_{0} \leqq 1$. Then $(2-\gamma) v_{0}=u_{1}+\gamma_{0} x_{0} \in L$, so that $v_{0} \in L$. This implies that $y_{0}=\alpha_{0} v_{0}+\beta_{0} x_{0} \in L$, which contradicts $y_{0} \in S-L$. The contradiction implies the theorem. 
THEOREM 4.8. Given the supposition of Theorem 4.6, there exists $z \in S$ such that $L z / 0=S$, where 0 is the additive identity of $S$.

Proof. By Theorem 4.7, $y_{0} / x_{0}$ is unbounded, so that for a given natural number $n$ there exists $v_{n} \in y_{0} / x_{0}$ such that $d\left(v_{n}, x_{0}\right) \geqq n$. Alternately, there exist $\alpha_{n}, \beta_{n}$, with $\alpha_{n}+\beta_{n}=1$ (and $\alpha_{n}>0$ ), such that $\alpha_{n} v_{n}+\beta_{n} x_{0}=y_{0}$. By Theorem 4.2, $\alpha_{n}=$ $d\left(x_{0}, y_{0}\right) / d\left(v_{n} x_{0}\right)$. Since $d\left(v_{n}, x_{0}\right) \geqq n, \alpha_{n}$ converges to 0 , and hence $\beta_{n}$ converges to 1 . Then $\beta_{n} x$ converges to $x$. By Theorem 4.1(b) $\alpha_{n} v_{n}$ must converge, say to $z$. We then have $z+x_{0}=y_{0}$.

We show that $L z / 0=S$. Observe that $u \in z / 0$ if and only if $u=\gamma z$, where $\gamma \geqq 1$. Let $z_{0} \in O y_{0} / x_{0}$, i.e. $z_{0}=\alpha_{0} u_{0}+\beta_{0} v_{0}$ and $\alpha v_{0}+\beta x_{0}=y_{0}$, where $\alpha+\beta=1=\alpha_{0}+\beta_{0}$. Then $\alpha v_{0}+\beta x_{0}=y_{0}=z+x_{0}, \alpha v_{0}=z+\alpha x_{0}, v_{0}=(1 / \alpha) z+x_{0}$. Substituting, $z_{0}=\alpha_{0} u_{0}$ $+\beta_{0}(x+(1 / \alpha) z)=\alpha_{0}\left(u_{0}+\left(\beta_{0} / \alpha_{0}\right) x_{0}\right)+\beta_{0}((1 / \alpha) z)$, with $u_{0}+\left(\beta_{0} / \alpha_{0}\right) x_{0} \in L$ and $(1 / \alpha) z$ $\in z / 0$. Hence, $z_{0} \in O z / 0$, and $O y_{0} / x_{0} \subset O z / 0$. Then $S=L y_{0} / x_{0} \subset L z / 0$, or $S=L z / 0$. This completes the theorem.

Actually $O z / 0=S$. For, let $z_{1} \in S$. There exists $\left\{z_{n}\right\} \subset O z / 0$ such that $z_{n}$ converges to $z$. Thus $z_{n}=\alpha_{n} u_{n}+\beta_{n}\left(\gamma_{n} z\right)$, where $u_{n} \in L, \gamma_{n} z \in z / 0, \gamma_{n} \geqq 1$, and $\alpha_{n}+\beta_{n}=1$. Since some subsequence of $\left\{\beta_{n}\right\}$ converges, we can say that $\left\{\beta_{n}\right\}$ converges, say to $\beta$. Suppose $\gamma_{n}$ is unbounded. Then $\left(1 / \gamma_{n}\right) z_{n}=\left(\alpha_{n} / \gamma_{n}\right) u_{n}+\beta_{n} z$, some subsequence of $\left(1 / \gamma_{n}\right) z_{n}$ converges to 0 , and $\beta_{n} z$ converges, so some subsequence of $\left\{\left(\alpha_{n} / \gamma_{n}\right) u_{n}\right\}$ converges, say to $u$. Then $0=u+\beta x$. But this implies $z \in I \subset L$, and $y_{0}=x_{0}+z \in L$. This contradicts $y_{0} \in S-L$. Thus $\left\{\gamma_{n}\right\}$ is bounded, so that without loss of generality we can say that $\gamma_{n}$ converges, say to $\gamma \geqq 1$. Again, $\alpha_{n} u_{n}$ must converge, say to $u$. We then have $z_{1}=u+\beta \gamma z$. If $\beta=1$, then $z_{1}=\frac{1}{2}(2 u)+\frac{1}{2}(2 \gamma z)$. If $\beta<1$, then $z_{1}=$ $\alpha((1 \alpha) u)+\beta(\gamma z)$. In either case, $z_{1} \in O z / 0$.

Note that from the conclusion of the above argument we can say that $z_{1} \in L z / 0$ if and only if $z_{1}=u+\gamma z$, where $u \in L, \gamma \geqq 0$. This will be used later.

Proof of Theorem 4.6. By Theorem 4.8, there is a $z$ such that $L z / 0=S$.

We show that if $x \in I$, then $L y_{0} / x_{1}=S$, where $x_{1}=x_{0}+x$.

Let $y \in O y_{0} / x_{0}$, so that $y=\alpha u+\beta v, \alpha_{0} v+\beta_{0} x_{0}=y_{0}$, with $\alpha+\beta=1=\alpha_{0}+\beta_{0}$. Since $x_{0}=x_{1}+(-x)$, we have $\alpha_{0} v+\left(x_{1}+(-x)\right)=y_{0}, \alpha_{0}\left(v+\left(\beta_{0} / \alpha_{0}\right)(-x)\right)+\beta_{0} x_{1}=y_{0}$. Also $y=\alpha u+\beta v+\left(\beta \beta_{0} / \alpha_{0}\right) x+\left(\beta \beta_{0} / \alpha_{0}\right)(-x)=\alpha\left(u+\left(\beta \beta_{0} / \alpha \alpha_{0}\right)(-x)\right)+\beta\left(v+\left(\beta_{0} / \alpha_{0}\right)(-x)\right)$. Since $-x \in I \subset L$, we have $u+\left(\beta \beta_{0} / \alpha \alpha_{0}\right)(-x) \in L$. We conclude that $y \in O y_{0} / x_{1}$, i.e. $O y_{0} / x_{0} \subset O y_{0} / x_{1}$. Thus $S=L y_{0} / x_{0}=L y_{0} / x_{1}$.

Therefore, if $I \neq\{0\}$, there is no unique $x_{0}$ such that $L y_{0} / x_{0}=S$.

To prove the converse, suppose there exist distinct points $x_{0}, x_{1} \in L$ such that $L y_{0} / x_{0}=L y_{1} / x_{1}=S$. By Theorem 4.8 (and its proof), there exist $z_{0}, z_{1} \in S$ such that $z_{0}+x_{0}=y_{0}$ and $z_{1}+x_{1}=y_{0}$, and $L z_{0} / 0=L z_{1} / 0=S$. From the remark immediately preceding this proof, there exist $u_{0} \in L$ and $\gamma_{1}$ such that $z_{1}=u_{0}+\gamma_{0} z_{0}$, and there exist $u_{1} \in L$ and $\gamma_{1}$ such that $z_{0}=u_{1}+\gamma_{0} z_{0}$, and there exist $u_{1} \in L$ and $\gamma_{1}$ such that $z_{0}=u_{1}+\gamma_{1} z_{1}$. Combining these,

$$
\begin{aligned}
z_{0} & =u_{1}+\gamma_{1}\left(u_{0}+\gamma_{0} z_{0}\right) \\
& =u_{1}+\gamma_{1} u_{0}+\gamma_{1} \gamma_{0} z_{0} .
\end{aligned}
$$


We cannot have $\gamma_{1} \gamma_{0}=0$, for otherwise, either $z_{0}$ or $z_{1}$ is in $L$, say $z_{0}$, implying that $S=L z_{0} / 0 \subset L$, and hence contradicting the assumption that $S$ has nonempty interior. Suppose $\gamma_{1} \gamma_{0}=1$. Then $0=u_{1}+\gamma_{1} u_{0}$, so that $u_{0}, u_{1} \in I$. Suppose $u_{1}=0$; then $u_{0}=0$ and $z_{0}=\gamma_{1} z_{1}$. Since $z_{0}+x_{0}=y_{0}=z_{1}+x_{1}$, we have $\gamma_{1} z_{1}+x_{0}=z_{1}+x_{1}$. If $\gamma_{1}=1$, then $x_{0}=x_{1}$, contradicting the assumption $x_{0} \neq x_{1}$. If $\gamma_{1}>1$, then $x_{1}=$ $\left(\gamma_{1}-1\right) z_{1}+x_{0}$. Since $x_{0}, x_{1} \in L$, by Theorem 4.5 , we have $\left(\gamma_{1}-1\right) z_{1} \in L$, or $z_{1} \in L$. But this would imply again that $L z_{1} / 0 \subset L$. If $\gamma_{1}<1$, a similar contradiction results. We conclude that $u_{0} \neq 0$, so that $I \neq\{0\}$, which was to be shown.

Now, suppose $\gamma_{0} \gamma_{1}>1$. Then $0=u_{1}+\gamma_{1} u_{0}+\left(\gamma_{0} \gamma_{1}-1\right) z_{0}$. We then have the nonzero $z_{0} \in I$, again implying $L z_{0} / 0 \subset L$.

Finally, suppose $\gamma_{0} \gamma_{1}<1$. Then $\left(1-\gamma_{1} \gamma_{0}\right) z_{0}=u_{1}+\gamma_{1} u_{0} \in L$. Again we have $z_{0} \in L$ implying that $L z_{0} / 0 \subset L$. This final contradiction shows that $\gamma_{1} \gamma_{0}=1$, and from above we see that $I \neq\{0\}$. This concludes the proof.

4. When is $L z / 0=S$ ? We now know that if $S$ is a Banach semilinear space with nonempty interior and there is a maximal linear subspace $L$ of $S$ and $x_{0} \in L$, $y_{0} \in S-L$ such that $L y_{0} / x_{0}=S$, then there must be a $z \in S$ such that, given $y$, there is $u \in L$ and $\gamma \geqq 0$ such that $y=u+\gamma z$.

Preliminary to showing necessary and sufficient conditions that $L z / 0=S$ for some $z$, we prove a uniqueness theorem.

As before, we assume that $S$ has nonempty interior, so that if $L z / 0=S$, then $z \notin L$.

THEOREM 4.9. If $L z / 0=S$ and $y=u_{1}+\gamma_{1} z=u_{2}+\gamma_{2} z, u_{1}, u_{2} \in L$, then $u_{1}=u_{2}$ and $\gamma_{1}=\gamma_{2}$.

Proof. Suppose $u_{1}+\gamma_{1} z=u_{2}+\gamma_{2} z$ and $\gamma_{1}>\gamma_{2}$. Then $u_{1}+\left(\gamma_{1}-\gamma_{2}\right) z=u_{2}$. Since $u_{1}$, $u_{2} \in L$, by Theorem $4.5\left(\gamma_{1}-\gamma_{2}\right) z \in L$, and hence $z \in L$. This contradicts $z \in L$. Similarly for $\gamma_{2}>\gamma_{1}$. Thus $\gamma_{1}=\gamma_{2}$. By cancellation, $u_{1}=u_{2}$.

THEOREM 4.10. Suppose $L$ is a maximal linear subspace of $\beta(S)$. Then $L z / 0=S$ if and only if $z \notin L$ and $z$ is in every maximal linear subspace $L^{\prime}$ of $\beta(S)$ distinct from $L$.

Proof. We show that if $z \notin L$ and $z \in L^{\prime}$ for every maximal linear subspace $L^{\prime}$ of $\beta(S)$ distinct from $L$, then $L z / 0=S$.

Let $y \in I(S)$, and consider $y+z$. By Theorem 4.3, if for every $x \in \beta(S), y \notin$ $[y+z, x]$, then $z \in I \subset L$, contradicting $z \notin L$. Then there is $x \in \beta(S)$ such that $y \in[y+z, x]$, i.e. $\alpha x+\beta(y+z)=y$, where $\alpha+\beta=1$, or $\alpha x+\beta z=\alpha y$. Clearly, $\alpha \neq 0$, so $y=x+(\beta / \alpha) z$. Suppose $x \notin L$. Every element of $\beta(S)$ belongs to a maximal linear subspace of $\beta(S)$ (proved by using Zorn's lemma), so that $x \in L^{\prime}$, for some maximal linear subspace $L^{\prime}$ of $\beta(S)$, which must be distinct from $L$, since $x \notin L$. But by hypothesis $z \in L^{\prime}$, hence $(\beta / \alpha) z \in L^{\prime}$. We then have $x+(\beta / \alpha) z=y \in L^{\prime}$. This contradicts $y \in I(S)$. Therefore, $x \in L$, so that $y=x+(\beta / \alpha) z$ implies $y \in O z / 0$. This 
shows that $I(S) \subset O z / 0$. By Theorem 2.4(c) $I(S)$ is dense in $S$. We conclude that $L z / 0$, the closure $O z / 0$, is $S$.

Conversely, suppose $L z / 0=S$, and let $L^{\prime}$ be a maximal linear subspace of $\beta(S)$ distinct from $L$. Now $z \notin L$, for otherwise $S=L z / 0 \subset L \subset \beta(S)$, implying $I(S)$ is empty. Let $x$ be an element of $L^{\prime}$ not in $L$. There exist $u \in L$ and $\gamma$ such that $x=u$ $+\gamma z$. Since $x \notin L, \gamma \neq 0$. The set of all $\alpha u+u^{\prime}$, where $\alpha \geqq 0$ and $u^{\prime} \in L^{\prime}$, is clearly linear. Suppose there is $\alpha_{0}>0$ and $u^{\prime} \in L^{\prime}$ such that $\alpha_{0} u+u^{\prime} \notin \beta(S)$. Then $\alpha_{0} u+$ $\alpha_{0}(\gamma z)+u^{\prime} \notin \beta(S)$, by Theorem 3.4(a). But $\alpha_{0} u+\alpha_{0}(\gamma z)+u^{\prime}=\alpha_{0}(u+\gamma z)+u^{\prime}=\alpha_{0} x+u^{\prime}$ $\in L^{\prime}$, since $\alpha x, u^{\prime} \in L^{\prime}$. This contradiction implies that $\alpha u+u^{\prime} \in \beta(S)$ for any $\alpha \geqq 0$, $u^{\prime} \in L^{\prime}$. Since $L^{\prime}$ is maximal we must have each $\alpha u+u^{\prime} \in L^{\prime}$; in particular, $u=1 \cdot u+0$ $\in L^{\prime}$. We have $x=u+\gamma z$ and $x, u \in L^{\prime}$. By Theorem 4.5, $\gamma z \in L^{\prime}$, and, since $\gamma \neq 0$, $z \in L^{\prime}$. This completes the proof.

EXAmPles. Consider the Banach semilinear space $C^{+}(0,1)$ of nonnegative continuous functions defined on $[0,1]$. The boundary of $C^{+}(0,1)$ consists of those $f$ such that $f(x)=0$, for some $x \in[0,1]$. Each maximal linear subspace of the boundary will be of the form $\left\{f: f\left(x_{0}\right)=0\right\}$, where $x_{0}$ is a fixed element of $[0,1]$. Let $L$ be such a subspace, corresponding to (say) $x_{0}$. Suppose $L z / 0=C^{+}(0,1)$, for some $z \in L$. Then $z\left(x_{0}\right) \neq 0$. By Theorem $4.10, z$ must be in every other maximal linear subspace of the boundary. As a consequence of this, $z(x)=0$ for all $x \neq x_{0}$, and $z\left(x_{0}\right) \neq 0$. Such a function is not continuous on $[0,1]$. Thus we see that $C^{+}(0,1)$ cannot be represented by any $L z / 0$, and hence by no $L y_{0} / x_{0}$.

Consider example (8). The boundary $\beta(S)$ consists of those sequences $\left\{x_{n}\right\}$ such that either $x_{n}=0$, for some $n$, or $\lim x_{n}=0$. The maximal linear subspaces of $\beta(S)$ are of the form $L_{n}=\left\{\left(x_{1} x_{2}, \ldots\right): x_{n}=0\right\}$, for $n$ some fixed positive integer, or $L_{0}=\left\{\left(x_{1} x_{2}, \ldots\right): \lim x_{n}=0\right\}$. For fixed positive integer $n$, let $z$ be the sequence with 1 in the $n$th term and 0 elsewhere. Then $z \in L_{m}$ for $m \neq n$ and $m=0,1, \ldots$ By Theorem 4.10, $L_{n} z / 0=S$. Now consider $L_{0}$. If $L_{0} z / 0=S$, then $z$ must belong to each $L_{n}, n=1,2, \ldots$, i.e. $z$ is identically 0 . Then $z \in L_{0}$. We conclude that $L_{0} z / 0 \neq S$ for any $z \in S$.

\section{Linear independence in semilinear spaces.}

Definition 4.4. Given a semilinear space $S$, a linear combination $\alpha_{1} x_{1}+\alpha_{2} x_{2}+$ $\cdots+\alpha_{n} x_{n}$, with $x_{i} \in S$ and $\alpha_{i} \geqq 0$, is said to be reduced if there do not exist $x_{i}, x_{j}$ such that $\alpha_{i} x_{i}+\alpha_{j} x_{j}=\beta x_{j}$, for some $\beta \geqq 0$. In other words, for no $x_{i}$ does $-x_{i}$ appear with nonzero coefficient if $\alpha_{i}>0$.

FACT. Every linear combination is equal to a reduced linear combination. We assume henceforth that all linear combinations have been reduced.

Definition 4.5. The set $\left\{x_{1}, x_{2}, \ldots, x_{n}\right\} \subset S$ is linearly independent provided that

$$
\alpha_{1} x_{1}+\alpha_{2} x_{2}+\cdots+\alpha_{n} x_{n}=\beta_{1} x_{1}+\beta_{2} x_{2}+\cdots+\beta_{n} x_{n}
$$

implies $\alpha_{i}=\beta_{i}$ for $i=1,2, \ldots, n$. 
Note that this definition allows $x$ and $-x$ to be included in a linearly independent set. This is necessary, since we cannot multiply by negative scalars.

Definition 4.6. A subset $A$ of $S$ is linearly independent if every finite subset of $A$ is linearly independent.

Given a linearly independent subset $A$ of $S$, Span $(A)$ will denote the set of all linear combinations of elements of $A$. If $\operatorname{Span}(A)=S, A$ is said to generate $S$.

THEOREM 4.11. Every semilinear space has a maximal linearly independent subset.

Proof. A routine application of Zorn's lemma.

Note. A semilinear space may have no maximal linearly independent subset which generates it. Consider $S=\left\{(x, y, z):\left(x^{2}+y^{2}\right)^{1 / 2} \leqq z\right\}$, with coordinatewise addition and scalar multiplication. Each maximal linearly independent subset of $S$ has exactly three elements, but the boundary $\left\{(x, y, z):\left(x^{2}+y^{2}\right)^{1 / 2}=z\right\}$ cannot be obtained in the span of such a set.

THEOREM 4.12. A linearly independent subset $A$ of the semilinear space $S$ is maximal if and only if, for each $x \in S$, there is $y \in \operatorname{Span}(A)$ such that $x+y \in \operatorname{Span}(A)$.

Proof. Suppose $A$ is maximal. Let $x \in S$. If $x \in \operatorname{Span}(A)$, then $x+x \in \operatorname{Span}(A)$, and the conclusion follows. Suppose $x \notin \operatorname{Span}(A)$. Then there exist scalars, $\alpha, \beta, \alpha_{1}, \ldots, \alpha_{n}, \beta_{1}, \ldots, \beta_{n}$, and $x_{1}, \ldots, x_{n} \in A$ such that $\alpha x+\alpha_{1} x_{1}+\cdots+\alpha_{n} x_{n}$ $=\beta x+\beta_{1} x_{1}+\cdots+\beta_{n} x_{n}$, but $\alpha_{i} \neq \beta_{i}$, for some $i$, or $\alpha \neq \beta$. In particular, $\alpha \neq \beta$, for otherwise we have by cancellation that $\alpha_{1} x_{1}+\cdots+\alpha_{n} x_{n}=\beta_{1} x_{1}+\cdots+\alpha x_{n}$ and $\alpha_{i} \neq \beta_{i}$ for some $i$. For simplicity assume $\alpha>\beta$. Then $(\alpha-\beta) x+\left(\alpha_{1} x_{1}+\cdots+\alpha_{n} x_{n}\right)$ $=\beta_{1} x_{1}+\cdots+\beta_{n} x_{n}$. Letting

$$
y=\frac{1}{(\alpha-\beta)}\left(\alpha_{1} x_{1}+\cdots+\alpha_{n} x_{n}\right)
$$

we have $x+y \in \operatorname{Span}(A)$.

Suppose now that $A$ is not maximal, i.e. there exists $x \notin \operatorname{Span}(A)$ such that $A \cup\{x\}$ is linearly independent. Suppose $x+y \in \operatorname{Span}(A)$ for some $y \in \operatorname{Span}(A)$. We then have $1 \cdot x+\alpha_{1} x_{1}+\cdots+\alpha_{n} x_{n}=0 \cdot x+\beta_{1} y_{1}+\cdots+\beta_{n} y_{n}$, or $1 \cdot x+\alpha_{1} x_{1}+$ $\alpha_{1} x_{1}+\cdots+\alpha_{n} x_{n}+0 \cdot y_{1}+\cdots+0 \cdot y_{n}=0 \cdot x+0 \cdot x_{1}+\cdots+0 \cdot x_{n}+\beta_{1} y_{1}+\cdots+$ $\beta_{n} y_{n}$. Since $1 \neq 0, A \cup\{x\}$ cannot be linearly independent. This contradiction concludes the proof.

THEOREM 4.13. If $S$ is a topological semilinear space, $A$ is a linearly independent subset of $S$ and Span $(A)$ has a nonempty topological interior, then $A$ is maximal.

Proof. Let $y_{0}$ be in the topological interior of $\operatorname{Span}(A)$. There is an open subset $V$ of $\operatorname{Span}(A)$ containing $x_{0}$. Let $x \in S$. If $x \in \operatorname{Span}(A)$, then $x+y_{0} \in \operatorname{Span}(A)$. Suppose $x \notin \operatorname{Span}(A)$. There must be $x_{0} \in\left[x, y_{0}\right] \cap V$ such that $x_{0} \neq x$ and $x_{0} \neq y_{0}$, i.e. $\alpha x+\beta y_{0} \in V$, where $\alpha+\beta=1$ and $\alpha>0, \beta>0$. Then $x+(\beta / \alpha) y_{0} \in \operatorname{Span}(A)$. We 
have therefore that if $x \in S$ there is $y \in \operatorname{Span}(A)$ such that $x+y \in \operatorname{Span}(A)$. By Theorem 4.12, $A$ is maximal.

THEOREM 4.14. If the maximal linearly independent subset $B$ of the Banach semilinear space $S$, with nonempty interior, generates $S$, then $B$ is not a subset of the boundary of $S$ if and only if every element of the boundary has an additive inverse.

Proof. If $\beta(S)=I$ and $B \subset \beta(S)$, then Span $(B) \subset \beta(S)$, contradicting $I(S) \neq \varnothing$. Thus if $\beta(S)=I$, then $B$ is not a subset of $\beta(S)$.

Conversely, suppose $B$ is not a subset of the boundary. Let $B^{\prime}=B \cap \beta(S)$. $B^{\prime}$ is not empty, for otherwise, by Theorem 3.4, Span $(S) \subset I(S)$. Given $x \in \beta(S), x=$ $\alpha_{1} x_{1}+\cdots+\alpha_{n} x_{n}$, with $x_{1}, \ldots, x_{n} \in B$ and $\alpha_{i} \neq 0$. No $x_{i}$ can be in $I(S)$, since, by Theorem 3.4, if some $x_{i} \in I(S)$, then $x \in I(S)$. Thus we have $x$ is a combination of elements of $B^{\prime}$, i.e. $\beta(S) \subset \operatorname{Span}\left(B^{\prime}\right)$. Let $x \in B \cap I(S)$. Let $y \in \beta(S), y \neq 0$. Suppose there is $x_{0} \in \beta(S)$ such that $x \in\left[x+y, x_{0}\right]$; then $\alpha x_{0}+\beta(x+y)=x, \alpha x_{0}+\beta y=\alpha x$. If $\alpha=0$, then $y=0$, so $\alpha>0$. Then $x=x_{0}+(\beta / \alpha) y$. Since $x_{0},(\beta / \alpha) y \in \beta(S) \subset \operatorname{Span}\left(B^{\prime}\right)$, we have $x=\alpha_{1} x_{1}+\cdots+\alpha_{n} x_{n}$, where $x_{1}, \ldots, x_{n} \in B^{\prime}$. But $x \notin B^{\prime}$, so $1 \cdot x+0 x_{1}+\cdots$ $+0 x_{n}=0 \cdot x+\alpha_{1} x_{1}+\cdots+\alpha_{n} x_{n}$, contradicting the linear independence of $B$. Thus if $x_{0} \in \beta(S)$, then $x \notin\left[x+y, x_{0}\right]$. By Theorem 4.3, $y \in I$. The conclusion is that $\beta(S)=I$, which completes the proof of the theorem.

COROLlary. Given the hypotheses of Theorem 4.14 and $B$ not a subset of $\beta(S)$, then $B \cap I(S)$ is a single point.

Proof. By Theorem $4.14, \beta(S)=I$, so that $\beta(S)$ is the only maximal linear subspace $L$ of $\beta(S)$. Suppose $x_{1}, x_{2} \in B \cap I(S)$ and $x_{1} \neq x_{2}$. By Theorem 4.10, $L x_{1} / 0$ $=S$. Then $x_{2}=u$ and $\gamma x_{1}$, where $u \in \beta(S)$. But then $u$ is a linear combination of elements of $\beta(S)$ and $x_{1}\left(\neq x_{2}\right)$. This gives that $x_{2} \in B$ is a linear combination of elements of $B$ distinct from $x_{2}$. The fact that $B$ is linearly independent rules this out. We cannot then have $x_{1}, x_{2} \in B \cap I(S)$ with $x_{1} \neq x_{2}$.

\section{Chapter 5. Semialgebras}

\section{Definitions and examples.}

Definition 5.1. The semilinear space $S$ is a semialgebra if there is an operation of multiplication (denoted by the usual notation) such that

(1) $(S, \cdot)$ is a semigroup.

(2) $(\alpha x)(\beta y)=(\alpha \beta)(x y), \alpha$ and $\beta$ nonnegative scalars, $x, y \in S$.

(3) $x(y+z)=x y+x z$ and $(y+z) x=y x+z x, x, y, z \in S$.

A topological semialgebra is a topological semilinear space which is a semialgebra such that multiplication is continuous.

A metric semialgebra is a metric semilinear space which is a semialgebra such that $d(x y, x z) \leqq d(y, z) d(x, 0)$ for any $x, y, z$.

A Banach semialgebra is a complete metric semialgebra.

Following Bourne, Bugenhagen, and others we have 
Definition 5.2. A subset $M$ of the semialgebra $S$ is an ideal provided that $M$ is a semialgebra and $a \in S, x \in M$ imply that $a x, x a \in M$.

Given $a, b \in S$, we say that $a$ is congruent to $b$ modulo the ideal $M$, denoted $a \equiv b(M)$, if there exist $m, n \in M$ such that $a+m=b+n$.

In the sequel we shall be concerned only with maximal proper ideals.

EXAMPLES. The natural modifications apply to examples (1) through (10). In addition, consider the following.

(11) Let $G=\left\{g_{1}, g_{2}, \ldots, g_{n}\right\}$ be a finite group. Let $L^{+}(G)$ be the set of all nonnegative real-valued functions defined on $G$. Given $f, g \in L^{+}(G)$ and $\alpha \geqq 0$, define $f+g, f g$, and $\alpha f$ by

$$
\begin{aligned}
(f+g)\left(g_{i}\right) & =f\left(g_{i}\right)+g\left(g_{i}\right), \\
(f g)\left(g_{k}\right) & =\sum_{i=1}^{n} f\left(g_{k} g_{i}^{-1}\right) g\left(g_{i}\right), \\
(\alpha f)\left(g_{i}\right) & =\alpha\left(f\left(g_{i}\right)\right) .
\end{aligned}
$$

With $d(f, g)=\sum_{i=1}^{n}\left|f\left(g_{i}\right)-g\left(g_{i}\right)\right|, L^{+}(G)$ is a Banach semialgebra. If $G$ is commutative, then so is $L^{+}(G)$. There is only one maximal ideal-the set of functions which are either identically 0 or nonzero on a nondegenerate subset of $G$.

2. The factor space $S / M$. Congruence modulo an ideal $M$ is easily seen to be an equivalence relation. We show transitivity. Suppose $a \equiv b(M)$. Then $a+m$ $=b+n$ and $b+p=c+q$, where $m, n, p, q \in M$. We have $a+(m+p)=(a+m)+p$ $=(b+n)+p=(b+p)+n=(c+q)+n=c+(q+n)$. Since $m+p, n+q \in M$, it follows that $a \equiv c(M)$.

Thus congruence modulo $M$ partitions $S$ into equivalent classes. We shall denote the equivalence class containing $x$ by $\bar{x}$ and the set of all such classes by $S / M$. The usual definitions of addition, multiplication, and scalar multiplication of equivalence classes carries over, and henceforth mention of $S / M$ will imply the availability of these operations.

Note. If $M$ is a maximal ideal, then $\overline{0}$ is an ideal containing $M$.

A curious fact is that $\overline{0}$ may not be proper, in which case we have $S / M=\{\overline{0}\}$. Consider $S=\{(x, y): 0 \leqq y \leqq x\}$, with coordinatewise operations. The only maximal ideal is $\{(x, y): 0 \leqq y<x\}$. Given $(x, y) \in S$, we have $(1,0) \in M$ and $(x, y)+(1,0) \in M$, so $(x, y) \equiv(0,0)(M)$, that is, $S=\overline{0}$. The single maximal ideal in example (11) also has this property.

Definition 5.3. An ideal $M$ will be called nonassimilating if there exists $x \in S$ such that $x+m \notin M$ for any $m \in M$.

If $M$ is any maximal ideal in a semialgebra $S$, consideration of the definitions of the operations on $S / M$ show immediately that $S / M$ is a semialgebra. If, in addition, $M$ is nonassimilating, we have that $S / M$ contains $\bar{x} \neq \overline{0}$. For there exists $x \in S$ such that $x \notin \overline{0}$; hence $\bar{x} \neq \overline{0}$. Also, since $M \subset \overline{0} \neq S$, we must have $M=\overline{0}$, by 
the maximality of $M$. Thus in case $M$ is nonassimilating, $M$ is the additive identity for $S / M$.

In some cases, $S / M$ is more well behaved than $S$, as we see in our first theorem on semialgebras.

THEOREM 5.1. Suppose $S$ is a commutative semialgebra and $M$ is a nonassimilating maximal ideal in $S$. Then the equation $\bar{a} \bar{x}=\bar{b}$, with $\bar{a} \neq \overline{0}$, has a solution $\bar{x}$ in $S / M$. In addition, $S / M$ has a multiplicative identity, and each nonzero element of $S / M$ has multiplicative inverse.

Proof. We first show that if $N$ and $P$ are ideals in $S$, the $(N, P)=\{n+p: n \in N$, $p \in P\}$ is an ideal. Clearly, $0 \in(N, P)$. Let $x, y \in(N, P)$, and $\alpha \geqq 0$. Then $x=n_{1}+p_{1}$, $y=n_{2}+p_{2}$, for some $n_{1}, n_{2} \in N$ and $p_{1}, p_{2} \in P$. We have $x+y=\left(n_{1}+n_{2}\right)+\left(p_{1}+p_{2}\right)$ $\in(N, P), x y=\left(n_{1}+p_{1}\right) n_{2}+\left(n_{1}+p_{1}\right) p_{2} \in(N, P), \alpha x=\alpha n_{1}+\alpha p_{1} \in(N, P), a x=a n_{1}+$ $a p_{1} \in(N, P)$, and $x a=n_{1} a+p_{1} a \in(N, P)$, for $a \in S$. Thus $(N, P)$ is an ideal.

We next show that, given $a \in S,(a)=\{a x: x \in S\}$ is an ideal. Let $y, z \in(a), b \in S$, and $\alpha \geqq 0$. Then $y=a x_{1}$ and $z=a x_{2}$, for some $x_{1}, x_{2} \in S$.

and

$$
\begin{aligned}
y+z & =a\left(x_{1}+x_{2}\right) \in(a), \\
y z & =\left(a x_{1}\right)\left(a x_{2}\right)=a\left(x_{1} a x_{2}\right) \in(a), \\
y & =\alpha\left(a x_{1}\right)=a\left(\alpha x_{1}\right) \in(a),
\end{aligned}
$$

$$
\begin{aligned}
& b y=b\left(a x_{1}\right)=a\left(b x_{1}\right) \in(a), \\
& y b=\left(a x_{1}\right) b=a\left(x_{1} b\right),
\end{aligned}
$$

using commutativity of multiplication.

We now prove the theorem.

Let $\bar{a}, \bar{b} \in S / M, \bar{a} \neq 0$. (a) is an ideal in $S$, so that $(M,(a))$ is an ideal. Since $M \subset(M,(a))$ and $a \notin M$, we have $(M,(a))=S$. Thus there exist $x \in S, m \in M$ such that $b=a x+m$, which implies that $\bar{b}=\bar{a} \bar{x}$.

Since $M$ is a nonassimilating ideal, there exists $\bar{a} \neq \overline{0}$. The equation $\bar{a} \bar{e}=\bar{a}$ holds for some $\bar{e}$. Let $\bar{b}$ be arbitrary. We have $\bar{a} \bar{x}=\bar{b}$, for some $\bar{x}$. Then $(\bar{a} \bar{e}) \bar{x}=\bar{a},(\bar{a} \bar{x}) \bar{e}$ $=\bar{a} \bar{x}$, so that $\bar{b}=\bar{b} \bar{e}$. Therefore $\bar{e}$ is a multiplicative identity.

Finally, given $\bar{a} \neq \overline{0}$, there is $\bar{x}$ such that $\bar{a} \bar{x}=\bar{e}$, so that $\bar{a}$ does indeed have a multiplicative inverse. This completes the proof of the theorem.

3. The boundary of a semialgebra. As a supplement to Theorem 3.1 , we have

THEOREM 5.2. If $S$ is a metric semialgebra, and $\lim x_{n}=x, \lim y_{n}=y$, then $\lim \left(x_{n} y_{n}\right)=x y$.

Proof. A routine application of the metric conditions of a metric semialgebra.

In the remainder of this section, we assume that $S$ is a Banach semialgebra with identity $e$ such that $e \in I(S)$ and $d(e, 0)=1$. 
THEOREM 5.3. If $x_{0} \in \beta(S)$ and $x \in S$, then $x x_{0} \in \beta(S)$.

Proof. Suppose $x x_{0} \in I(S)$. We are assuming $e \in I(S)$, so $x+e \in I(S)$, by Theorem 3.4(a). By definition of $I(S)$, there exists $x^{\prime} \in S, \alpha>0, \beta>0$, with $\alpha+\beta=1$, such that $\alpha(x+e)+\beta x^{\prime}=e$. Then $\alpha x+\beta x^{\prime}=\beta e$, and $\alpha\left(x x_{0}\right)+\beta\left(x^{\prime} x_{0}\right)=\beta x_{0}$. Since $x x_{0} \in I(S)$ and $\alpha>0$, Theorem 3.4(b) implies that $\beta x_{0} \in I(S)$, and hence $x_{0} \in I(S)$. The theorem follows.

THEOREM 5.4. Suppose $M$ is a maximal nonassimilating ideal in $S$ and there exists $x$ such that $\bar{x} \cap \beta(S)$ is empty. Then $\beta(S)=M=I$.

Proof. We show $\beta(S) \subset M$. Suppose $x_{0} \in \beta(S)-M$. Since $M$ is maximal and nonassimilating, there exists $a \in S, m \in M$ such that $x=a x_{0}+m$, by Theorem 5.1. Then $a x_{0} \in \bar{x}$, and, by Theorem 5.3, $a x_{0} \in \beta(S)$. Thus $\bar{x} \cap \beta(S) \neq \varnothing$. This contradiction implies $\beta(S) \subset M$.

We next show $M \subset I$. Choose $m \in M, \neq 0$. We must show $m$ has an additive inverse. Consider $x+m$, and suppose there is $x_{0} \in \beta(S)$ such that $x \in\left[x+m, x_{0}\right]$, i.e. there exist $\alpha>0, \beta>0$, with $\alpha+\beta=1$, such that $\alpha x_{0}+\beta(x+m)=x$. Then $\alpha x_{0}+\beta m=\alpha x$, and $x_{0}+(\beta / \alpha) m=x$. Then $x_{0} \in \bar{x}$, and $\bar{x} \cap \beta(S) \neq \varnothing$, a contradiction. Thus, given $x_{0} \in \beta(S), x \notin\left[x+m, x_{0}\right]$. By Theorem 4.3, $m$ has an additive inverse.

By Theorem 3.4, $I \subset \beta(S)$. Then $M \subset I \subset \beta(S) \subset M$ implies $\beta(S)=M=I$.

4. A theorem from Banach algebras. An element $x$ in the semialgebra $S$ with identity $e$ is regular if it has a multiplicative inverse, and singular otherwise.

Let $S$ be a Banach semialgebra with identity $e \in I(S)$ such that $d(e, 0)=1$.

Note. No element of $\beta(S)$ can be regular, since by Theorem 5.3, $x_{0} \in \beta(S)$ implies $x x_{0} \in \beta(S)$, for any $x \in S$, and $e \notin \beta(S)$.

THEOREM 5.5. If $d(a, e)<1, a \in S$, then $a$ is regular.

Proof. By Definition 2.3 we have $d(x+y, 0) \leqq d(x+y, y)+d(y, 0)=d(x, 0)$ $+d(y, 0)$, and $d(x y, 0) \leqq d(x, 0) d(y, 0)$. Using these facts and mathematical induction, we have

$$
d\left(\sum_{i=n}^{m} x^{i}, 0\right) \leqq \sum_{i=n}^{m}[d(x, 0)]^{i}
$$

From this and the completeness of $S$, we have that if $d(x, 0)<1$, then $\sum_{n=0}^{\infty} x^{n}$ converges, letting $x^{0}=e$.

Suppose $d(a, e)<1$. Since $e \in I(S), e \notin \beta(S)$, so that there exist $x \in S, \alpha>0$, $\beta<0$, with $\alpha+\beta=1$, such that $\alpha x+\beta a=e$. Now

$$
\begin{aligned}
d(\alpha x, 0) & =d(\alpha x+\beta a, \alpha a)=d(e, \beta a) \leqq d(e, \beta e)+d(\beta e, \beta a) \\
& =d(\alpha e, 0)+\beta d(a, e)=\alpha d(e, 0)+\beta d(a, e)<\alpha+\beta=1 .
\end{aligned}
$$

Therefore $\sum_{n=0}(\alpha x)^{n}$ converges. 


$$
\begin{aligned}
& \alpha x+\beta a=e \\
& (\alpha x)^{2}+\beta a(\alpha x)=\alpha x \\
& (\alpha x)^{3}+\beta a(\alpha x)^{2}=(\alpha x)^{2} \\
& (\alpha x)^{n+1}+\beta a(\alpha x)^{n}=(\alpha x)^{n} \\
& e+\sum_{i=1}^{n+1}(\alpha x)^{i}+\beta a \sum_{i=0}^{n}(\alpha x)^{i}=\sum_{i=0}^{n}(\alpha x)^{i}+e \\
& \sum_{i=0}^{n+1}(\alpha x)^{i}+\beta a \sum_{i=0}^{n}(\alpha x)^{i}=e+\sum_{i=0}^{n}(\alpha x)^{i} \\
& \sum_{n=0}^{\infty}(\alpha x)^{n}+\beta a \sum_{n=0}^{\infty}(\alpha x)^{n}=e+\sum_{n=0}^{\infty}(\alpha x)^{n} .
\end{aligned}
$$

By cancellativity of addition,

$$
a\left(\beta \sum_{n=0}^{\infty}(\alpha x)^{n}\right)=e .
$$

Therefore, $a$ is regular and its multiplicative inverse is $\beta \sum_{n=0}^{\infty}(\alpha x)^{n}$.

Corollary. If $r$ is regular and $d(x, r)<1 / d\left(r^{-1}, 0\right)$, then $x$ is regular. Hence the set of regular elements is open.

Proof. $d\left(x r^{-1}, e\right)=d\left(x r^{-1}, r r^{-1}\right) \leqq d(x, r) d\left(r^{-1}, 0\right)<1$. This implies $x r^{-1}$ is regular, so that $x$ is regular.

Theorem 5.5 implies the same result for Banach algebras, since every Banach algebra is equal to its interior.

The last results settle the question of the existence of maximal ideals for Banach semialgebras with the assumed conditions. Each maximal ideal must be a subset of the set of singular elements, which is closed, by the preceding corollary. Using arguments paralleling those from Banach algebras, we conclude that each maximal ideal is closed and that each singular element is contained in such a maximal ideal.

In the context of this section we can prove a result related to the work of Bonsall mentioned in the introduction.

A closed semialgebra of continuous functions in $C(X)$ is clearly a Banach semialgebra. A closed semialgebra $S$ of $C(X)$ is said to be of the type $n(n \geqq 0)$ provided $f \in S$ implies $f^{n} /(1+f) \in S$. Bonsall has succeeded in characterizing closed semialgebras of types 0 and 1 . We state and prove a result on type 0 semialgebras.

THEOREM 5.6. Suppose $S$ is a closed semialgebra in $C(X)$. Then $S$ is of type 0 if and only if $1 \in S$ and $\beta(S)$ is the set of singular elements of $S$.

Proof. Recall $S$ is of type 0 if $f \in S$ implies $1 /(1+f) \in S$. 
Suppose $1 \in S$ and $\beta(S)$ is the set of singular elements. The $I(S)$ is the set of regular elements. Since 1 is regular, we have $1 \in I(S)$. Given $f \in S$, we have $1+f$ $\in I(S)$, using Theorem 3.4(a). Hence $1+f$ has a multiplicative inverse $1 /(1+f) \in S$, and $S$ is of type 0 .

Conversely, suppose $f$ is of type 0 . Since $0 \in S, 1=1 /(1+0) \in S$. Bonsall has proved that $f, g \in S, f \geqq g$, implies $f-g \in S$. Given $f \in S$, choose $\alpha$ such that $0<\alpha<1$ and $\alpha f \geqq 1$. Let $\beta=1-\alpha$ and $g=(1-\alpha f) / \beta$. We have $g \in S$ and $\alpha f+\beta g=1$. Thus, given $f \neq 1$, there exist $g \neq 1$ and $\alpha>0, \beta>0$, with $\alpha+\beta=1$, such that $\alpha f+\beta g=1$. This implies $1 \notin \beta(S)$; hence $1 \in I(S)$. We have $1 \in I(S), d(1,0)=1$, and $S$ a Banach semialgebra. Then, by Theorem 5.3, $x \in \beta(S)$ implies $x x_{0} \in \beta(S)$ for any $x_{0} \in S$. Then, given $x \in \beta(S), x$ cannot be regular, since otherwise $x x_{0}=1 \in I(S)$, for some $x_{0} \in S$. This means $\beta(S)$ is a subset of the set of singular elements of $S$. Now suppose there is a singular element $f$ of $S$ which is not in $\beta(S)$. Then there exist $g \neq 1$ and $\alpha>0, \beta>0$, with $\alpha+\beta=1$, such that $\alpha(1)+\beta g=f$, or $1+(\beta / \alpha) g=(1 / \alpha) f$. Since $S$ is of type $0,1+(\beta / \alpha) f$ is regular. Hence $f$ is regular. This contradiction implies $\beta(S)$ is the set of singular elements of $S$, and the theorem is proved.

The condition $1 \in S$ was needed in Theorem 5.6. For consider $X=\{(x, 0): x \geqq 0\}$, with coordinatewise operations. $X$ is a Banach semialgebra, $(1,0)$ is a multiplicative identity. We consider $X$ as a semialgebra in the space $C(E)$, with $E$ a two point set, i.e. $C(E)$ is the plane. In this context, $\beta(X)=\{0\}$, which is the set of singular elements relative to $(1,0)$, but $X$ is not type 0 in $S$. Note that $(1,1)$, the multiplicative identity of $C(E)$, is not in $X$.

5. The nondegeneracy of $\beta(S)$. We have seen that $0 \in \beta(S)$, for any topological semialgebra $S$ which is not an algebra $(N \neq \varnothing)$. When is $\beta(S)=\{0\}$ ? We give a partial answer.

Let $S=\{(x, y): x=0=y$ or $x>0$ and $y>0\}$ with coordinatewise operations. $S$ is a metric semialgebra and $\beta(S)=\{0\} . S$ is not complete.

THEOREM 5.7. Suppose $S$ is a Banach semialgebra, with $N \neq \varnothing$. Then $\beta(S)=\{0\}$ if and only if $S=\left\{\alpha x_{0}: \alpha \geqq 0\right\}$, for some fixed $x_{0} \in S$.

Proof. It is clear that $S=\left\{\alpha x_{0}: \alpha \geqq 0\right\}$ implies $\beta(S)=\{0\}$.

Suppose $S$ is not $\left\{\alpha x_{0}: \alpha \geqq 0\right\}$, for any $x_{0} \in S$, but that $\beta(S)=\{0\}$. Then, given $y \in S$, there exists $x \in S$ such that $x \notin[0, x+y]$. Since 0 is the only element of $\beta(S)$, the hypotheses of Theorem 4.3 are satisfied, so that $y \in I$. Therefore $S=I$, which contradicts the assumption $N \neq \varnothing$.

\section{REFERENCES}

1. E. J. Barbeau, Semi-algebras that are lower semi-lattices, Pacific J. Math. 18 (1966), 1-7. MR 33 \#6360.

2. - The principal semi-algebra in a Banach algebra, Trans. Amer. Math. Soc. 120 (1965), 1-16. MR 32 \#371.

3. - Maximal closed semialgebras of continuous nonnegative functions on a locally compact Hausdorff space, Notices Amer. Math. Soc. 14 (1967), 410. Abstract \#67T-276. 
4. F. F. Bonsall, Semi-algebras of continuous functions, Proc. Internat. Sympos. Linear Spaces (Jerusalem 1960), Jerusalem Academic Press, Jerusalem and Pergamon, Oxford, 1961, pp. 101-114. MR 27 \#4064.

5. - Locally compact semi-algebras, Proc. London Math. Soc. (3) 13 (1963), 51-70. MR $26 \# 615$.

6. - On the representation of cones and semi-algebras with given generators, Proc. London Math. Soc. (3) 15 (1965), 39-60. MR 30 \# 440.

7. F. F. Bonsall and B. J. Tomiuk, The semi-algebra generated by a compact linear operator, Proc. Edinburgh Math. Soc. (2) 14 (1964/65), 177-196. MR 32 \#1557.

8. S. Bourne, On normed semi-algebras, Studia Math. 21 (1961/62), 45-54. MR 25 \#4378.

9. —_ On Banach*-semialgebras, Studia Math. 21 (1961/62), 207-214. MR 25 \#4379.

10. A. C. Baker and J. W. Baker, Duality of topological semigroups with involution, J. London Math. Soc. 44 (1969), 251-260.

11. - A note on duality of topological semigroups, J. London Math. Soc. 44 (1969), 261-264.

12. K. H. Hofmann, Uber lokalkompakte positive Halbkörper, Math. Ann. 151 (1963), 262-271. MR 27 \#4213.

13. M. A. Kaashoek, Locally compact semialgebras and spectral theory, Nieuw Arch. Wisk. (3) 17 (1969), 111.

14. K. Keimel, Lokal Kompakte Kegelhalbgruppen und deren Einbettung in topologische Vektorraüme, Math. Z. 96 (1967), 405-428.

15. A. L. Peressini, Ordered topological vector spaces, Harper \& Row, New York, 1967. MR 37 \#3315.

16. H. H. Schaefer, Topological vector spaces, Macmillan, New York, 1966. MR 33 \#1689.

17. B. J. Tomiuk, Left completely continuous semialgebras, Fund. Math. 64 (1969), 123-140.

\section{Georgia State University,} Atlanta, Georgia 